\title{
Comparison of AutoDock and Glide towards the Discovery of PPAR Agonists
}

\author{
Rajyalakshmi Gaddipati, Gitesh K. Raikundalia, and Michael L. Mathai
}

\begin{abstract}
Peroxisome Proliferator Activated Receptors are lipid-sensors and regulate energy metabolism. The agonists of PPARs are of interest to the pharmaceutical industry since they regulate the expression of genes associated with diseases like cancer, diabetes, atherosclerosis and obesity. Synthetic agonists are more likely to cause side effects. Hence eight naturally occuring lipid ligands (tocotrienol $\alpha, \beta, \gamma$ and $\delta$, DHA, EPA, 2-Arachidonyl Glycerol and Anandamide) were tested for their ability to act as the agonists of PPARs. DHA and EPA were identified as the dual agonists of PPAR $\alpha$ and $\gamma$. DHA and EPA have beneficial health effects in the treatment of cancer, obesity and inflammatory diseases. Two different docking methods Autodock and Glide were performed to compare their suitability for PPARs. Interestingly in both the docking programs the ligands have occupied the same binding pocket confirming the selection of active site. Autodock yielded better results than Glide for PPAR $\alpha$ and $\gamma$ whereas the performance of Glide was better in case of PPAR $\delta$.
\end{abstract}

Index Terms-Agonists of PPARs, autodock, glide, omega 3 fatty acids.

\section{INTRODUCTION}

Peroxisome Proliferator Activated Receptors (PPARs) are members of the nuclear receptor family. They exist in three is forms: PPAR $\alpha, \operatorname{PPAR} \beta / \delta$ and PPAR $\gamma$. The pharmaceutical industry is paying more attention to the research of PPAR agonists, since PPARs regulate the gene expression of different diseases like cancer, diabetes, atherosclerosis and obesity [1]. PPARs regulate energy metabolism by each carrying out a unique function and serve as therapeutic targets to treat obesity and homeostasis [2]. PPAR $\alpha$ and $\gamma$ are expressed in liver and adipose tissue, respectively. PPAR $\delta$ is expressed throughout the body and low levels in liver. PPAR $\gamma$ acts as a molecular target for the anti-diabetic drugs thiazolidinediones (TZDs). Recent research suggests that PPAR $\gamma$ has a therapeutic potential to treat inflammatory diseases and certain cancers [3]. PPAR $\alpha$ is the molecular target for lipid-lowering fibrate drugs. The metabolic regulatory role of PPAR $\delta$ is recently recognized and clinical trials for PPAR $\delta$ agonists are underway [2].

PPARs are lipid-sensors that can be activated by both dietary fatty acids and their metabolic derivatives in the body [2].

Manuscript received August 25, 2013; revised November 23, 2013

Rajyalakshmi Gaddipati and Gitesh K. Raikundalia are with the College of Engineering and Science, Victoria University, Melbourne, Australia (e-mail: gitesh.raikundalia@vu.edu.au).

Michael L. Mathai is with the College of Health and Biomedicine, Victoria University, Melbourne, Australia (e-mail: michael.mathai@vu.edu.au).
The long and medium chain unsaturated fatty acids are the most abundant ligands of PPARs [4]. Considering these facts, three classes of natural lipid ligands are selected to test their ability as potential agonists of PPARs. Four isomers of tocotrienols: tocotrienol $\alpha, \beta, \gamma$ and $\delta$ belong to class one of lipids. Omega 3 Fatty acids (Docosahexaenoic acid, DHA and Eicosapentanoic acid, EPA) are considered under class two of lipid ligands. Endocannabinoids (2-Arachidonyl glycerol and Anandamide) are the third class of lipid ligands. The above ligands are selected since fatty acids and eicosanoids are natural agonists of PPARs [5]. Moreover the synthetic ligands cause more side effects than the natural ligands [6].

All the eight lipid ligands used in the current study are chemically active and medicinally significant. Tocotrienols were demonstrated to have positive health effects on bone health, brain health, blood sugar metabolism and cancer [7]. Animal studies suggest that omega 3 fatty acids exert protective effects against breast, colon and prostate cancers. In patients with colorectal cancer DHA and EPA decrease cell proliferation and maintain the balance between colonic cell proliferation and apoptosis [8]. Endocannabinoids are used in the treatment of obesity and as an aid in the cessation of smoking [9]. Endocannabinoids are capable of reducing inflammation, cell proliferation and cell survival and hence can be used in cancer treatment [10].

In the current study the binding mechanism of PPARs with the above mentioned eight lipid ligands was studied through molecular docking. Two different docking tools, AutoDock (http://autodock.scripps.edu) and Glide [11], were used to identify which docking method works better with the target proteins and lipids. The binding of PPARs with the above mentioned eight lipid ligands was not performed before either insilico or invitro.

This paper is organized as follows. Section II describes the literature review carried as part of the current research. Methods performed were mentioned in Section III. The findings from the current research work are included in Section IV. Section V and Section VI are about the conclusion and future work.

\section{LITERATURE REVIEW}

The limitations of currently used PPAR based drugs have laid a foundation for the current docking study of finding the new agonists that activate PPARs. Anti-diabetic and hypolidemic drugs are PPAR-based. However their use is contraindicated in patients with high lipid levels [12]. For example widely used TZDs result in some side effects like obesity and developing the risk of cardiovascular disease [13]. PPAR $\alpha$-based lipid-lowering fibrate drugs are limited 
in their efficacy due to restricted selectivity [14].

Therefore there is a need to find potential agonists of PPAR $\gamma$ and $\alpha$ that cause few or no side effects. Moreover the dual agonists of PPAR $\alpha$ and $\gamma$ are of interest to the pharmaceutical industry. Though a similar docking study was conducted before for the binding affinities of PPARs, the study was limited to DHA [12].

The current docking study is also useful in finding the potential molecular target of PPAR $\delta$. Because of number of docking tools available today, it is always a challenge to select the suitable technique for a target protein. Hence two widely used docking techniques AutoDock and Glide were compared to find out the best suitable docking tool for PPARs. The current molecular docking study is novel as it proposes the dual agonists of PPAR $\alpha$ and $\gamma$ and agonists of $\operatorname{PPAR} \delta$. Furthermore, the present research is unique as the suitable docking tool for PPARs was identified.

\section{METHODS}

PPAR $\alpha, \beta$ and $\gamma$ are tested for their binding affinities with eight lipid ligands $(\alpha, \beta, \gamma$ and $\delta$ tocotrienols, DHA, EPA, $2 \mathrm{AG}$ and Anandamide) using two different docking methodsAutoDock and Glide.

\section{A. Ligand Preparation}

The three dimensional structures of all the eight ligands were downloaded from Pubchem website (http://pubchem.ncbi.nlm.nih.gov/) as the website has a huge collection of ligand structures. The ligand structures are minimized with Chimera (http://www.cgl.ucsf.edu/chimera) to perform AutoDock. Hydrogens are added using AutoDock tools. The LigPrep module of Maestro v9.2 in the Schrodinger suite of tools [15] is used for the ligand preparation to perform Glide docking.

\section{B. Protein Preparation and Receptor Grid Generation}

The three dimensional structures of PPAR $\alpha, \beta$ and $\gamma$ were downloaded from Protein Data Bank website (www.pdb.org). PDB codes 3FEI, 3GZ9 and 3FEJ were used for the crystal structures of PPAR $\alpha, \beta$ and $\gamma$ respectively. Refining the crystal structures by removing crystal ligands and water molecules is performed using Chimera prior to using AutoDock. The amino acids Cys 275, Cys 276, Met 330 and Met 355 were considered as the active site for the protein PPAR $\alpha$ [16]. For PPAR $\delta$ the combination of amino acids His 323, His 449, Tyr 473, Cys 285 and Thr 288 is the active site [17]. Met 364, Cys 285, Met 348 and Gly 284 are the active site amino acids for PPAR $\gamma$ [16]. The receptor grids are generated for the active site amino acids.

To perform Glide docking the protein structures were prepared using the Protein Preparation Wizard of Maestro. The crystal ligand CTM is used for the receptor grid generation of both PPAR $\alpha$ and $\gamma$, whereas for PPAR $\delta$ the crystal ligand is D32. Except for the bridging water molecules rest of them were deleted during the minimization of proteins.

\section{Validation of Docking Studies}

Redocking is performed as a validation method for all docked protein-ligand complexes. The three dimensional structures of crystal ligands were obtained by removing the crystal ligand from protein crystallographic complexes. The crystal ligands are then redocked with the three PPAR proteins using AutoDock and Glide. Root Mean Square Deviation (RMSD) values between crystal ligand and predicted confirmations were calculated using Pymol software (http://www.pymol.org/).

\section{Docking Studies}

Docking is performed by using two different methods: AutoDock and Glide. PPAR $\alpha, \beta$ and $\gamma$ were docked with the above mentioned eight lipid ligands. Genetic algorithm GA with 10 iterative runs and Lamarckian genetic algorithm were selected to perform AutoDock. The results of AutoDock were analyzed using VMD software (http://www.ks.uiuc.edu/Research/vmd/). Extra Precision (XP) mode of Glide docking is performed with the receptor grid files and prepared ligand structures. Ligplot software (http://www.ebi.ac.uk/thornton-srv/software/LIGPLOT) is used to study the bonded interactions of PPARs with lipid ligands.

\section{RESUlTS AND DisCUSSION}

\section{A. Redocking as a Docking Validation Method}

Redocking is the most significant validation method to evaluate the accuracy of the docking procedure [18]. Redocking determines how closely the lowest binding energy pose resembles the experimental binding mode determined by $X$-ray crystallography. In the current study, AutoDock and Glide were validated by removing the crystal ligand (CTM for PPAR $\alpha$ and $\gamma$ and D32 for PPAR $\delta$ ) from the binding site and redocking it to the binding site of PPAR $\alpha$ (PDB code: 3FEI), PPAR $\gamma$ (PDB code: 3FEJ) and PPAR $\delta$ (PDB code: 3GZ9). The alignment of PPAR $\alpha, \operatorname{PPAR} \beta$ and PPAR $\gamma$ with crystal ligand were depicted in Fig. 1, Fig. 2 and Fig. 3 respectively where the redocked ligand is shown in red color.
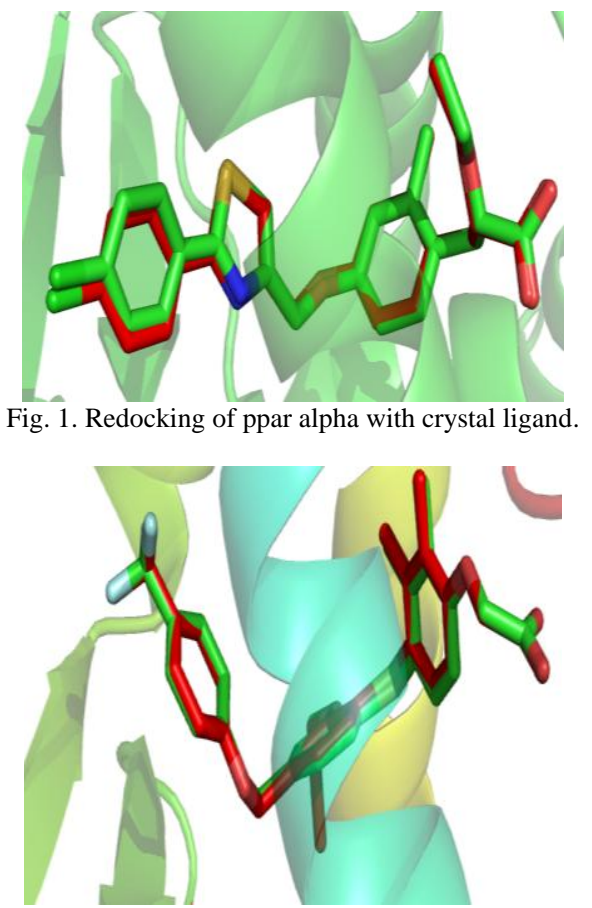

Fig. 2. Redocking of ppar delta with crystal ligand. 


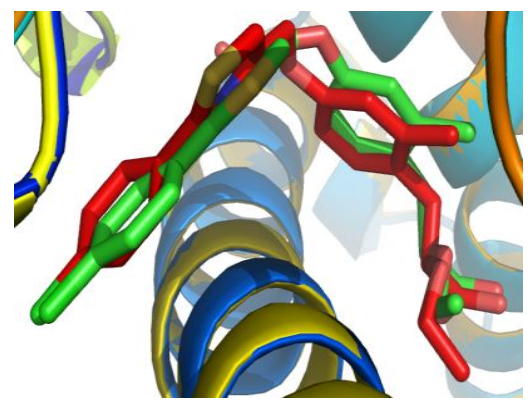

Fig. 3. Redocking of PPAR gamma with crystal ligand.

The crystal ligand is removed from the binding site and superimposed with X-ray crystal structures of PPARs and RMSD were calculated. RMSD between the predicted confirmation and the observed binding mode for all three isoforms of PPARs is less than $2 \mathrm{~A}^{0}$ and is similar in both AutoDock and Glide (Table I). RMSD values between crystal and predicted confirmation is an indicator of whether or not correct docking was obtained from a particular docking method [19]. RMSD less than $2 \mathrm{~A}^{0}$ is the cut-off of correct docking, perhaps because the resolution in an X-ray crystallography is often about $2 \mathrm{~A}^{0}$ and higher precision than the resolution of crystal structure analysis is not meaningful [18]. Therefore AutoDock and Glide docking performed in the current study were considered to be successful as the RMSD between crystal and predicted confirmation is less than $2 \mathrm{~A}^{0}$ (Table I).

TABLE I: RMSD VALUES OF CRYSTAL LIGAND

\begin{tabular}{ccccc}
\hline $\begin{array}{l}\text { Serial } \\
\text { Number }\end{array}$ & Protein & $\begin{array}{l}\text { Crystal } \\
\text { Ligand }\end{array}$ & $\begin{array}{l}\text { RMSD } \\
\text { AutoDock }\end{array}$ & $\begin{array}{l}\text { RMSD } \\
\text { Glide }\end{array}$ \\
\hline 1. & PPAR $\alpha$ & CTM & 0.160 & 0.169 \\
2. & PPAR $\delta$ & D32 & 0.203 & 0.123 \\
3. & PPAR $\gamma$ & CTM & 0.165 & 0.162 \\
\hline
\end{tabular}

\section{B. Docking Analysis}

The research on agonists of PPARs is significant as PPARs play key roles in the regulation of energy homoeostasis and inflammation [20]. The agonists of PPARs are currently used therapeutically. The dual agonists of PPAR $\alpha$ and $\gamma$ are used in the treatment of diabetes and dyslipidemia and hence are of high importance. The anti-diabetic drugs thiazolidinediones (TZDs) use PPAR $\gamma$ as a molecular target [13]. However TZDs cause side effects like obesity and cardiovascular diseases [13]. A potential therapeutic target of PPAR $\delta$ is under investigation [20]. Considering the medicinal importance of PPARs in the treatment of different diseases like diabetes, atherosclerosis and cancer they are docked with eight lipid ligands to test their potentiality as PPAR agonists.

Moreover, the lipid ligands used in the current study are also medicinally significant. For example, tocotrienols are chemically active and are used in the treatment of breast cancer [7]. Omega 3 fatty acids (DHA and EPA) play an important role in the pathophysiology and treatment of bipolar disorder [21]. Endocannabinoids can reduce inflammation, cell proliferation and cell survival and hence can be used in the treatment of cancer [10]. These facts imply that the current docking experiment is significant as a way of designing PPAR-based drugs.

The binding energies of PPAR $\alpha, \beta$ and $\gamma$, achieved through using AutoDock and Glide were mentioned in Table II. Out of all the eight ligands tested in the current docking study DHA has expressed strong binding with both PPAR $\alpha$ and $\gamma$. Therefore DHA can be considered as a dual agonist of PPAR $\alpha$ and $\gamma$. Interestingly, DHA has shown strong binding affinity than the crystal ligand CTM. CTM was considered as crystal ligand as it was identified as a potential dual agonist of PPAR $\alpha$ and $\gamma$ in previous studies. In an experiment PPARs were tested for their binding affinity with 26 ligands where CTM was identified as strong affinity dual agonist of PPAR $\alpha$ and $\gamma$ [16]. Apart from CTM, DHA was also proved to have strong affinity with the TZDs of rosiglitazone [12] and pioglitazone [22]. The current in silico experiment shows that DHA has strong affinity with PPAR $\alpha$ and $\gamma$ and so can be considered as potential natural dual agonist of PPAR $\alpha$ and $\gamma$.

Comparatively, omega 3 fatty acids (DHA and EPA) have shown strong binding with PPAR $\alpha$ and $\gamma$ (Table II). It was also proved in another experiment that DHA activates PPAR $\alpha$ and $\gamma$ genes [22]. Furthermore, all four isoforms of tocotrienols can be considered as dual agonists of PPAR $\alpha$ and $\gamma$ since they exhibited strong affinity than the crystal ligand CTM (Table II).

TABLE II: BINDING ENERGIES OF PPARS IN AUTODOCK AND GLIDE

\begin{tabular}{llcc}
\hline Protein & Ligand & $\begin{array}{l}\text { AutoDock-Lowest } \\
\text { binding energy } \\
\text { (Kcal/m) }\end{array}$ & $\begin{array}{l}\text { Glide } \\
\text { Score(K } \\
\text { cal/m) }\end{array}$ \\
\hline PPAR $\alpha$ & DHA & -11.5 & -10.2 \\
PPAR $\alpha$ & EPA & -10.1 & -9.3 \\
PPAR $\alpha$ & 2 AG & -7.69 & -8.0 \\
PPAR $\alpha$ & Anandamide & -6.57 & -5.6 \\
PPAR $\alpha$ & $\alpha$ tocotrienol & -9.87 & -7.1 \\
PPAR $\alpha$ & $\beta$ tocotrienol & -9.98 & -7.5 \\
PPAR $\alpha$ & $\gamma$ tocotrienol & -8.44 & -7.3 \\
PPAR $\alpha$ & $\delta$ tocotrienol & -9.53 & -7.5 \\
PPAR $\alpha$ & CTM & -7.72 & -7.5 \\
PPAR $\beta$ & DHA & -11.40 & -15.8 \\
PPAR $\beta$ & EPA & -10.93 & -14.8 \\
PPAR $\beta$ & 2 AG & -9.22 & -10.8 \\
PPAR $\beta$ & Anandamide & -8.67 & -9.7 \\
PPAR $\beta$ & $\alpha$ tocotrienol & -9.20 & -9.7 \\
PPAR $\beta$ & $\beta$ tocotrienol & -9.97 & -9.3 \\
PPAR $\beta$ & $\gamma$ tocotrienol & -9.09 & -9.8 \\
PPAR $\beta$ & $\delta$ tocotrienol & -9.31 & -7.7 \\
PPAR $\beta$ & D32-Cryslig & -10.2 & -11.7 \\
PPAR $\gamma$ & DHA & -11.71 & -10.3 \\
PPAR $\gamma$ & EPA & -10.22 & -9.4 \\
PPAR $\gamma$ & 2 AG & -7.74 & -7.7 \\
PPAR $\gamma$ & Anandamide & -6.29 & -5.2 \\
PPAR $\gamma$ & $\alpha$ tocotrienol & -8.93 & -8.2 \\
PPAR $\gamma$ & $\beta$ tocotrienol & -8.95 & -8.4 \\
PPAR $\gamma$ & $\gamma$ tocotrienol & -9.66 & -8.5 \\
PPAR $\gamma$ & $\delta$ tocotrienol & -9.68 & -8.5 \\
PPAR $\gamma$ & CTM & -8.0 & -8.1 \\
\hline & & &
\end{tabular}

Endocannabinoids have shown poor affinity with PPAR $\alpha$ and $\gamma$ compared to omega 3 fatty acids and tocotrienols. PPAR $\alpha$ and $\gamma$ are similar in their binding with the lipid ligands used in the current docking study. PPAR $\delta$ also showed strong binding with omega 3 fatty acids than with the crystal ligand D32. However, unlike PPAR $\alpha$ and $\gamma$, PPAR $\delta$ has poor affinity with tocotrienols than with the crystal ligand D32. Similar to PPAR $\alpha$ and $\gamma$, PPAR $\delta$ expressed strong affinity with the crystal ligand D32 than with endocannabinoids. 


\section{Binding Mode of Lowest Binding Energy Poses}

Interestingly in both AutoDock and Glide docking tools the ligands have occupied the same binding pocket indicating the accuracy of the active site selected to generate the receptor grid. The hydrogen bonded and hydrophobic interactions of DHA with PPAR $\alpha, \operatorname{PPAR} \beta$ and PPAR $\gamma$ were represented in Fig. 4, Fig. 5 and Fig. 6 respectively where hydrogen bonds and bond distances are represented in green colors and hydrophobic amino acids in purple color.

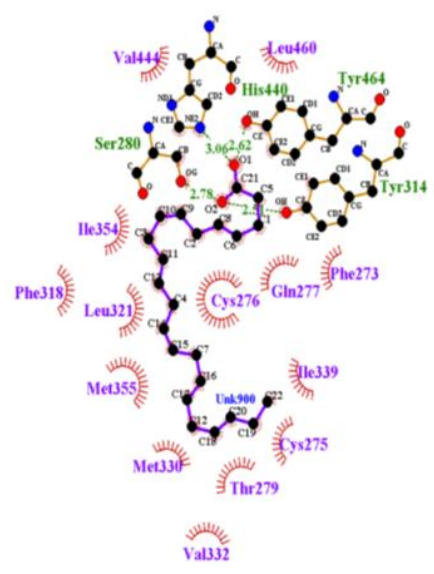

PPARa-DHA

Fig. 4. Bonded interactions of PPAR alpha with DHA.

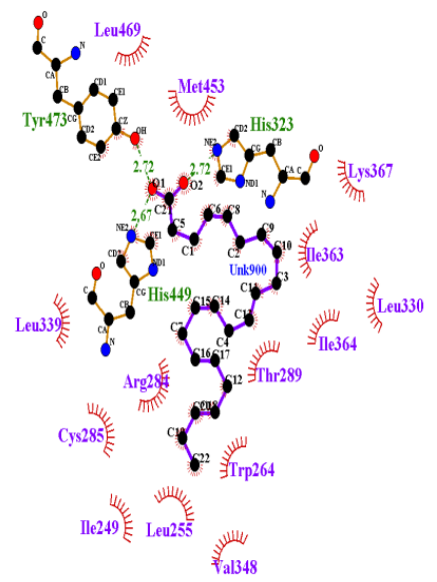

PPARd-DHA

Fig. 5. Bonded interactions of PPAR delta with DHA.

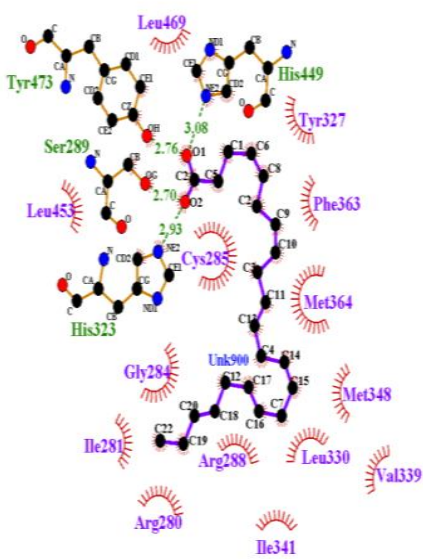

PPARg_DHA

Fig. 6. Bonded Interactions of PPAR gamma with DHA.
TABLE III: INTERACTING AMINO ACIDS OF PPARS IN AUTODOCK AND GLIDE

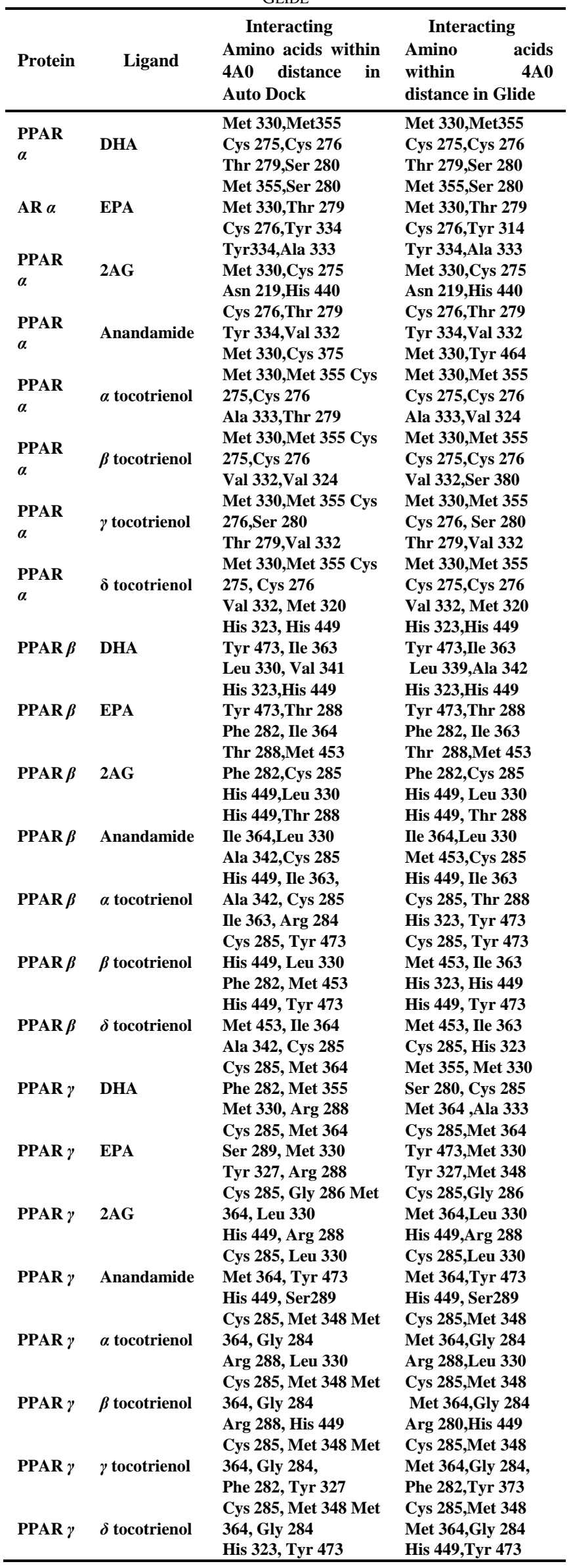

PPAR $\alpha$ has formed hydrophobic interactions with the active site amino acids Cys 275, Cys 276, Met 330 and Met 
355 (Fig. 4). Oxygen (O) 1 located on carbon (C) 21 of DHA has formed two hydrogen bonds with His 440 and Tyr 364 with bond distances $3.06 \mathrm{~A}^{0}$ and $2.62 \mathrm{~A}^{0}$ respectively. Ser 280 and Tyr 314 have formed hydrogen bonds with $\mathrm{O} 2$ of $\mathrm{C} 21$ on DHA with bond distances $2.78 \mathrm{~A}^{0}$ and $2.51 \mathrm{~A}^{0}$ as shown in Fig. 4.

In the case of PPAR $\delta$, the active site amino acids His 323, His 449 and Tyr 473 have formed hydrogen bonds with the ligand (Fig. 5). His 323 and Tyr 473 have formed two hydrogen bonds with $\mathrm{O} 1$ located on $\mathrm{C} 21$ of DHA with bond distances 2.67A0 and 2.72A0 respectively (Fig. 5). O2 on C21 of DHA formed a hydrogen bond with His 323 in distance of 2.72A0. Cys 285, Thr 289, Ile 363, Thr 279 have formed hydrophobic interactions with the ligand. The active site amino acids Cys 285, Met 348, met 364 and Gly 284 of PPAR $\gamma$ have formed hydrophobic interactions with the ligand. His 449 and Tyr 473 of PPAR $\gamma$ have formed two hydrogen bonds with $\mathrm{O} 1$ located on $\mathrm{C} 21$ of DHA with a bond distance of 3.08A0 and 2.76A0 as shown in Fig. 6. Two more hydrogen bonds were observed with $\mathrm{O} 2$ on C21 of DHA with bond a distance of 2.93 from His 323 and 2.70 from Ser 289 (Fig. 6).

To conclude, omega 3 fatty acids are potential agonists for all three isoforms of PPARs. In comparison, tocotrienols are the dual agonists of PPAR $\alpha$ and $\gamma$.

\section{Comparison of AutoDock and Glide}

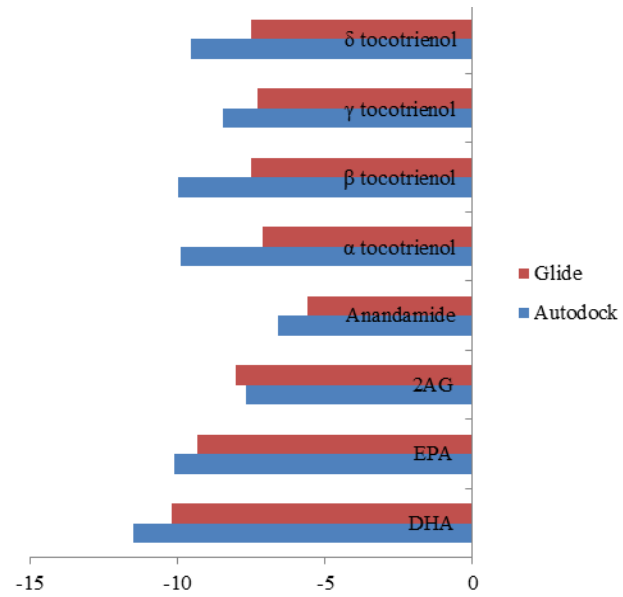

Fig. 7. Comparision of binding energies of PPAR alpha in AutoDock and glide.

AutoDock and Glide have generated similar binding energies for all the three isoforms of PPARs with all the eight lipid ligands docked in the current study (Table I). In order to compare the similarity between both AutoDock and Glide, the interacting amino acids within $4 \mathrm{~A} 0$ distance from the ligand were shown in Table III.

The common interacting amino acids of both the docking methods were represented in bold.

The active site is confirmed for PPARs since the ligands occupied the same binding site in both AutoDock and Glide programs. Though AutoDock and Glide uses different scoring functions, they resulted in generating the similar binding energies. AutoDock uses empirical scoring function and Glide uses XP scoring function. Apart from the slight difference in the binding energies generated from AutoDock and Glide docking tools, the interaction of protein with ligand is similar in both the methods. Using two different methods of docking and resulting in similar interaction of target proteins with ligand indicates the accuracy of the current insilico experiment.

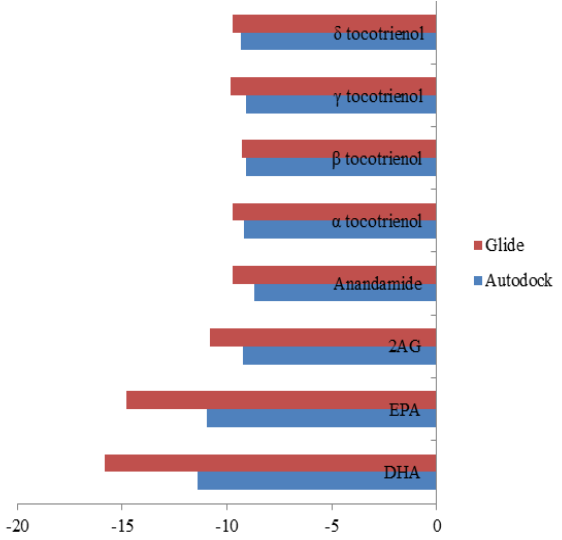

Fig. 8. Comparision of binding energies of PPAR delta in AutoDock and glide.

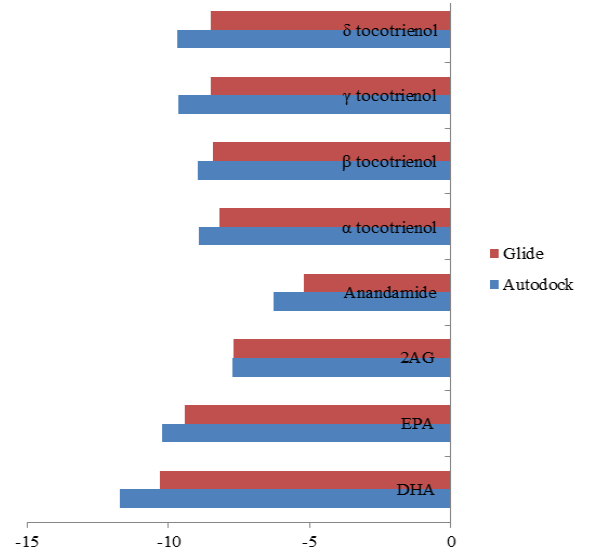

Fig. 9. Comparision of binding energies of PPAR gamma in AutoDock and glide.

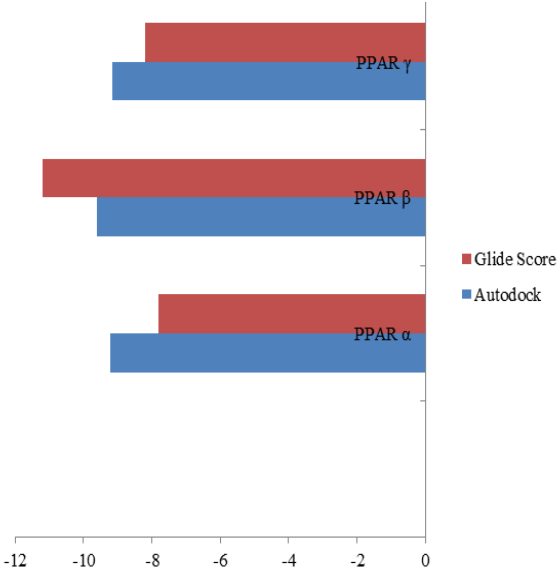

Fig. 10. Comparision of binding energies of PPARs in AutoDock and glide.

AutoDock worked well for the proteins PPAR $\alpha$ and $\gamma$ whereas Glide yielded better results for PPAR $\delta$. To compare the efficiency of both AutoDock and Glide the bar graphs were drawn with the help of binding energies obtained from both the docking methods. AutoDock is efficient for both PPAR $\alpha$ and $\gamma$ while Glide is an efficient tool for PPAR $\delta$ (Fig. 10). Individual efficiency of AutoDock and Glide for the interaction of eight lipid ligands with $\operatorname{PPAR} \alpha, \beta$ and $\gamma$ is shown in Fig. 7, Fig. 8 and Fig. 9 respectively. 


\section{CONCLUSIONS}

Redocking is performed as a validation method of AutoDock and Glide. Omega 3 fatty acids were observed as the dual agonists of PPAR $\alpha$ and $\gamma$. Tocotrienols and omega 3 fatty acids have shown strong binding affinity with PPAR $\alpha$ and $\gamma$ than with the crystal ligand CTM. PPAR $\delta$ has shown strong binding affinity with omega 3 fatty acids compared to the other two classes of lipid ligands. AutoDock was identified as a suitable docking program for PPAR $\alpha$ and $\gamma$ whereas for PPAR $\delta$ Glide is the suitable docking program. In both AutoDock and Glide the ligands have occupied the same binding pocket generating the similar docking poses.

\section{FUTURE WORK}

Omega 3 fatty acids and tocotrienols can further be tested invitro for their ability to act as the dual agonists of PPAR $\alpha$ and $\gamma$. The dual agonists of PPAR $\alpha$ and $\gamma$ are significant in anti-diabetic and anti-cancer therapy. For the research of PPAR $\delta$ agonists omega 3 fatty acids can be considered and further tested.

\section{ACKNOWLEDGMENT}

The authors would like to thank Victoria University and Melbourne University for providing the facilities required for the study.

\section{REFERENCES}

[1] T. Oyama and K. Toyota et al., "Adaptability and selectivity of human peroxisome proliferator-activated receptor (PPAR) pan agonists revealed from crystal structures," Acta Crystallographica Section, vol. 65, no. 8. pp. 786-795, 2009.

[2] B. G. Evans and Y. X. Wang, "PPARs and the complex journey to obesity," Nature Medicine, vol. 10, no. 4, pp.355-361, 2004.

[3] G. J. Murphy and J. C. Holder, "PPAR-gamma agonists: therapeutic role in diabetes, inflammation and cancer," Trends in pharmacological sciences, vol. 21, no. 12, pp. 469-474, 2000.

[4] F. Fang and Z. Kang et al., "Vitamin E tocotrienols improve insulin sensitivity through activating peroxisome proliferator-activated receptors," Molecular Nutrition \& Food Research, vol. 54, no. 3, pp. 345-352, 2010.

[5] S. A. Kliewer and S. S. Sundseth et al., "Fatty acids and eicosanoids regulate gene expression through direct interactions with peroxisome proliferator-activated receptors $\alpha$ and $\gamma$, " in Proc. the National Academy of Sciences, 1997, vol. 94, no. 9, pp. 4318-4323.

[6] R. W. Nesto and D. Bell et al., "Thiazolidinedione use, fluid retention, and congestive heart failure: A consensus statement from the American heart association and American diabetes association," Circulation, vol. 108, no. 23, pp. 2941-2948, 2003.

[7] B. B. Aggarwal and C. Sundaram et al., "Tocotrienols, the vitamin E of the 21st century: It's potential against cancer and other chronic diseases," Biochemical Pharmacology, vol. 80, no. 11, pp. 1613-1631, 2010 .

[8] A. P. Simopoulos, "Omega-3 fatty acids and cancer," Indoor and Built Environment, vol. 12, no. 6, pp. 405-412, 2003.

[9] K. O. Jonsson and S. Holt et al., "The endocannabinoid system: Current pharmacological research and therapeutic possibilities," Basic \& Clinical Pharmacology \& Toxicology, vol. 98, no. 2, pp. 124-134, 2006.

[10] S. Sarfaraz and V. M. Adhami et al., "Cannabinoids for cancer treatment: Progress and promise," Cancer Research, vol. 68, no. 2, pp. 339-342, 2008.

[11] Glide, version 5.7, Schrödinger, LLC, New York, NY, 2011.

[12] O. A. B. S. M. Gani and I. Sylte, "Molecular recognition of Docosahexaenoic acid by peroxisome proliferator-activated receptors and retinoid-X receptor $\alpha$, , Journal of Molecular Graphics and Modelling, vol. 27, no. 2, pp 217-224, 2008.

[13] R. R. V. Malapaka and S. Khoo et al., "Identification and mechanism of 10-carbon fatty acid as modulating ligand of peroxisome proliferator-activated receptors," Journal of Biological Chemistry, vol. 287, no. 1, pp. 183-195, 2012.

[14] M. L. Sierra and V. Beneton et al., "Substituted 2-[(4-Aminomethyl) phenoxy]-2-methylpropionic acid PPAR $\alpha$ agonists. 1. discovery of a novel series of potent HDLc raising agents," Journal of Medicinal Chemistry, vol. 50, no. 4, pp. 685-695, 2007.

[15] Maestro, version 9.2, Schrödinger, LLC, New York, NY, 2011.

[16] U. Grether and A. Bénardeau et al., " Design and biological evaluation of novel, balanced dual PPAR $\alpha / \gamma$ agonists," ChemMedChem. vol. 4, no. 6, pp. 951-956, 2009.

[17] R. V Connors and Z. Wang et al., "Identification of a PPAR $\delta$ agonist with partial agonistic activity on PPAR $\gamma$, , Bioorganic \& Medicinal Chemistry Letters, vol. 19, no. 13, pp. 3550-3554, 2009.

[18] S. Tripathi and C. Selvaraj et al., "Molecular docking, QPLD, and ADME prediction studies on HIV-1 integrase leads," Medicinal Chemistry Research, vol. 21, no. 12, pp. 4239-4251, 2012.

[19] R. T. Kroemer and A. Vulpetti et al., "Assessment of docking poses: Interactions-Based Accuracy Classification (IBAC) versus crystal structure deviations," Journal of Chemical Information and Computer Sciences, vol. 44, no. 3, pp. 871-881, 2004.

[20] B. Staels and J. C. Fruchart, "Therapeutic roles of peroxisome proliferator-activated receptor agonists," Diabetes, vol. 54, no. 8, pp. 2460-2470, 2005.

[21] A. L. Stoll and C. A. Locke et al., "Omega-3 fatty acids and bipolar disorder: a review," Prostaglandins, Leukotrienes and Essential Fatty Acids, vol. 60, no. 5-6, pp. 329-337, 1999.

[22] T. Itoh and I. Murota et al., "Synthesis of docosahexaenoic acid derivatives designed as novel PPAR $\gamma$ agonists and antidiabetic agents," Bioorganic \& Medicinal Chemistry, vol. 14, no. 1, pp. 98-108, 2006.

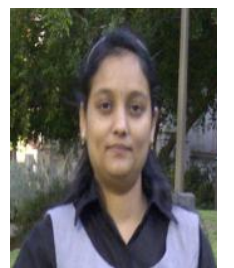

Rajyalakshmi Gaddipati is a $\mathrm{PhD}$ student in bioinformatics in the College of Engineering and Science at Victoria University, Australia. She received her B.Sc. in biochemistry from Andhra University, India in 200, M.Sc. in biochemistry from the Andhra University, India in 2003, and MPhil in biochemistry from the Bharathidasan University, India in 2008, Graduate Diploma in biotechnology from the Swinburne University, Australia.

She has worked as a lecturer in biochemistry in Aurora Degree College and Sujatha Post Graduate College both in India. She is currently doing a $\mathrm{PhD}$ in Melbourne. She has presented one of her research papers at the ICEAS 2012 international conference held in Beijing, China. Her current research interests are computer aided drug design, drug discovery, molecular docking and molecular dynamic simulations.

Gitesh K. Raikundalia is a senior lecturer in computer science in the College of Engineering and Science at Victoria University, Australia. He received his B.Ec. in economics, operations research and computer science from The University of Sydney, Australia, in 1991, M.Comp from The University of Newcastle, Australia, in 1994 and Ph.D. in information technology from Bond University, Australia, in 1998.

He has held academic positions previously at Southern Cross University and Swinburne University of Technology, both in Australia. His current position is based in Melbourne, Australia. He is currently acting as Discipline Group Leader (Networks and Security) and has been Academic Coordinator (Research \& Research Training) in the former School of Engineering and Science. He has 85 publications that have attracted nearly 350 citations (excluding self-citations) and over $\$ 850,000$ in grant funding. His research interests include Health Informatics, Computer Supported Cooperative Work and Software Engineering. He is an Associate Member of the Australian Computer Society.

Michael Mathai is an associate professor at the College of Health and Biomedicine at Victoria University. He received his BSc. (Hons) and PhD (1992) from the University of Melbourne. He has held research positions at the Florey Institute of Mental Health Research (Australia) and the Max-Planck Institute of Physiology and Clinical Research (Germany). He is currently Course Leader for the Nutritional Therapy at Victoria University. $\mathrm{He}$ has more than 100 publications with >1000 citations, and over $\$ 1.2$ million in grant funding. His research interests include molecular mechanisms involved in micronutrient supplementation, the central control of homeostasis and improving public health via advances in food labeling. $\mathrm{He}$ is a member of the Nutrition Society of Australia and the American Physiology Society. 